\title{
Determinates of Childhood Pneumonia and Diarrhea with Special Emphasis to Exclusive Breastfeeding in North Achefer District, Northwest Ethiopia: A Case Control Study
}

\author{
Molla Gedefaw ${ }^{1 *}$, Resom Berhe ${ }^{2}$ \\ ${ }^{1}$ GAMBY College of Medical Science, Bahir Dar, Ethiopia \\ ${ }^{2}$ Health Education and Behavioral Sciences Unit, Institute of Public Health, University of Gondar, Gondar, \\ Ethiopia \\ Email: ${ }^{*}$ mollagedefaw@yahoo.com, resomberhe@yahoo.com
}

Received 5 March 2015; accepted 4 May 2015; published 12 May 2015

Copyright (C) 2015 by authors and Scientific Research Publishing Inc.

This work is licensed under the Creative Commons Attribution International License (CC BY).

http://creativecommons.org/licenses/by/4.0/

(c) (i) Open Access

\section{Abstract}

Studies showed that exclusive breast feeding reduced infant morbidity and mortality. In low income countries such as Ethiopia where under-five mortality is very high, the role of exclusively breastfeeding could be even more critical. However, studies assessing the place of exclusive breast freeing in the prevention of childhood illnesses in our area are scarce. The aim of the study was to identify determinant factors of childhood pneumonia and diarrhoea. An institution based case control study was conducted in Achefer District in July, 2012. The cases were 122 children of 7 - 24 months old who had repeated attack of diarrhoea or pneumonia over three months prior to the survey while controls were 122 children who visited well baby clinic for vaccination. Data were collected by using pre-tested and structured questionnaire, and analysed using SPSS version 16 for windows. Logistic regression was performed, and strength of associations was estimated using odds ratio and $95 \%$ confidence interval. About $83 \%$ of the controls and only $12.3 \%$ of the cases were exclusively breast fed. Children who were exclusively breast fed were 83 times less likely to develop pneumonia or diarrhea than those who were not exclusively fed. Marital status, monthly income, prelacteal feeding, and late initiation of breast feeding were found to have statistically significant association with childhood diarrhea and pneumonia. This study brought local evidence that exclusive breast feeding had a protective effect against common childhood infectious dis-

\footnotetext{
"Corresponding author.
} 
eases-pneumonia and diarrhoea-in the study area. Therefore, culture sensitive and plausible health education is recommended to strengthen exclusive breast feeding practices in order to decrease mortality and morbidity of infants and children from pneumonia and diarrhoea.

\section{Keywords}

\section{Exclusive Breast Feeding, Determinant Factors, Childhood Pneumonia, Diarrhea, Ethiopia}

\section{Background}

Exclusive breastfeeding refers to feeding an infant only with breast milk for six months [1]. World Health Organization (WHO) has identified exclusive breastfeeding as the optimal feeding regime for children. United Nations Children's Fund (UNICEF) suggests also that breast milk alone is the ideal nourishment for infants in their first six months of life. It is capable of providing all of the nutrients, such as vitamins and minerals [2].

Breastfeeding is the ideal method suited for the physiological as well as psychological needs of an infant. Hence, it is widely recognized as method of infant feeding worldwide [1] [2].

Despite its multitudes of advantages, poor breastfeeding practices are widespread. Reviews of studies from developing countries show that infants who are not exclusively breastfed are 6 to 10 times more likely to die in the first months of life than infants who are exclusively breastfed [3]. According to Lancet Nutrition Series 2008, babies who do not breastfeed are, generally, more than 14 times more likely to die from diarrhoea or respiratory infections than babies who are exclusively breastfed in the first six months [4]. Partial breastfeeding does not seem to provide this protective effect against infections in infancy [4] [5].

It is estimated that sub-optimal breastfeeding in the first six months of life results in 1.4 million deaths and $10 \%$ of the disease burden in children younger than 5 years of age [3]. Researches indicate that breast-feeding protects against acute lower respiratory tract infection (pneumonia) because of breast milk's unique anti-infective properties [6]. According to Cesar (2012) [6], infants under 2 months old who are not breastfed are six times more likely to die from diarrhea or acute respiratory infections than those who are breastfed.

Ethiopia is one of the countries with very high infant, and child morbidity, and mortality. Diarrhoea and pneumonia are the most important causes of childhood morbidity, and mortality. However, despite multiple benefits, exclusive breast feeding is not widely practiced. Moreover, its benefits in relation to prevention of childhood diseases such as pneumonia and diarrhoea are not well studied in our areas. Therefore, the finding of this study is of theoretical and practical significance especially for researchers and program managers for future planning.

\section{Methods}

An institutional-based unmatched case-control study was conducted. The study was conducted in North Achefer district, Northwest Ethiopia. According to the housing and population census conducted in 2006 during the restructuring of Achefer District, the total population size of North Achefer Woreda was 213, 920. The district has seven health centres, two higher clinics, 24 health posts, 24 private and one non-governmental health institution. The study was conducted in three government health centres. They were selected based on patient load, and presence of trained staff to treat children based on integrated management of neonates and childhood illness (IMNCI) guideline.

Target population for this study were children aged $7-24$ months. The cases were those children visited health institutions because of repeated attack of diarrhoea or pneumonia. The controls were normal children who were brought to well baby clinic for vaccination. The study was conducted in July, 2012. Children who were from outside the catchment area of the selected health centres were excluded from the study.

The sample size was calculated using sample size calculation for two population proportion formula "difference between population proportions". Accordingly, including $10 \%$ non response rate, the minimum total sample size required was 122 case and 122 controls. Epi-info soft ware was used to calculate sample size.

Cases were those children who received care for pneumonia, and diarrhoea at the selected health centres, and 
registered in the IMNCI registration book at least once in a month over three months prior to the survey. Controls were children who were brought to the selected health centres for vaccination purpose, and had no history of diarrhoea, and pneumonia over three months prior to the survey.

The questionnaire was pre-tested on 30 mothers having children aged 7 - 24 months, who visited the health institution. Then corrections were made based on the pre-test findings. Supervision was conducted by two supervisors and the principal investigator. At the end of each day, interviewers were submitted all completed questionnaire and each completed questionnaire were checked for accuracy and completeness. Moreover, questionnaire of forty respondents (5.0\%) were administered by supervisors and the principal investigator. Data was entered into computer, and SPSS version 16.0 was used for statistical analysis. About $10 \%$ of the data was double entered to assure the accuracy of data entry. Binary logistic regression was fitted, and possible association and statistical significance were assessed using odds ratio at $95 \%$ confidence interval.

Ethical clearance was obtained from the Ethical Review Committee of Bahirdar University. Permission and support letter was provided to each selected health institutions. The purpose of the study was explained to the study participants and a verbal consent was obtained prior to the interview. Confidentiality of responses was insured throughout the research process.

\section{Results}

\section{Socio-Demographic Characteristics of Study Participants}

A total of 244 children met the inclusion criteria. Table 1 displays characteristics of children. As shown in the table, mean age was $8.97 \pm 3.65$ and $7.97 \pm 1.44$ months for cases and controls respectively. About $53.3 \%$ and $46.7 \%$ children from case and controls were females, respectively. About $75 \%$, and $95 \%$ of children were between 7 - 12 months of age in cases, and control, respectively.

About 72 (59.06\%) and 81 (66.39\%) of mothers of cases and controls were housewives. More than $80 \%$ of mothers were able to read and write, and $41 \%$, and $33.6 \%$ of mothers of cases and controls were employed. Nearly $70 \%$ of mothers were less than 30 years old. About $63 \%$, and $35 \%$ of mothers claimed to have more than 3 children (Table 2).

Table 3 displays factors which had statistically significant association with occurrence of pneumonia, and diarrhea in this study. As shown in the table, low birth weight, exclusive breast feeding, monthly income of mothers, and prelacteal feeding were found to be determinants of pneumonia, and diarrhea. Children with history of low birth weight, prelacteal feeding, and not exclusively breast feed were 12, 16, and 83 times more likely to cases. Moreover, children born to mothers whose monthly income were less than 850 Birr were nearly two times more likely to be cases than those whose mothers monthly income was higher than 850 Birr. However, in this study educational status of mothers fall below the level of statistical significance (Table 3).

Table 1. Age and sex distribution of children 7 - 24 months old in cases and controls in North Achefer District, Northwest Ethiopia, July 2012.

\begin{tabular}{ccc}
\hline Age (in month) & Cases (\%) & Controls (\%) \\
\hline Mean (SD) & $8.97(3.65)$ & $7.97(1.44)$ \\
Age in month & $91(74.5 \%)$ & $117(95.9 \%)$ \\
$7-12$ & $31(25.5 \%)$ & $5(4.1 \%)$ \\
$13-24$ & \\
Sex & $79(64.8)$ & $43(35.2)$ \\
Male & $65(53.3)$ & $57(46.7)$ \\
Female &
\end{tabular}


Table 2. Socio-demographic characteristics of mothers of case and control North Achefer District, Northwest Ethiopia, 2012.

\begin{tabular}{lcc}
\hline \multicolumn{1}{c}{ Characteristics $(\mathrm{N}=244)$} & Cases $(\%)(\mathrm{n}=122)$ & Controls $(\%)(\mathrm{n}=122)$ \\
\hline $\begin{array}{l}\text { Maternal age (in years) } \\
<30\end{array}$ & $85(69.71 \%)$ & $83(68.04 \%)$ \\
$\geq 30$ & $37(30.29 \%)$ & $39(31.96 \%)$ \\
Education (mother) & $20(16.39 \%)$ & $12(9.8 \%)$ \\
$\quad$ Unable to read and write & $102(83.61 \%)$ & $110(90.2 \%)$ \\
Able to read and write & & $79(64.8 \%)$ \\
No. of children & $45(36.88 \%)$ & $43(35.2 \%)$ \\
$\leq 3$ & $77(63.11 \%)$ & $121(99.2 \%)$ \\
$>3$ & & $1(0.8 \%)$ \\
Ethnicity & $110(92.1 \%)$ & $81(66.4 \%)$ \\
Amhara & $12(9.8 \%)$ & \\
Others & & \\
Occupation of mothers & $50(41 \%)$ & $(33.6 \%)$ \\
Employed & $72(59 \%)$ & \\
Unemployed & & \\
\hline
\end{tabular}

Table 3. Determinant of pneumonia, and diarrhea among 7 - 24 months old children, North Achefer District, Northwest, Ethiopia, 2012.

\begin{tabular}{|c|c|c|c|c|c|}
\hline Variable & Categories & Case & Control & AOR & $95 \%$ CI \\
\hline \multirow{2}{*}{ Birth weight $<2500$} & Yes & 35 & 6 & 12.4 & $1.9-79.1^{*}$ \\
\hline & No & 87 & 116 & 1 & \\
\hline \multirow{2}{*}{ Pre-lacteal feeds given } & Yes & 107 & 21 & 15.9 & $2.55-99.48^{*}$ \\
\hline & No & 15 & 101 & 1 & \\
\hline \multirow{2}{*}{ Exclusive breast-feeding } & Yes & 15 & 101 & 1 & \\
\hline & No & 107 & 21 & 83.3 & $26.31-250^{*}$ \\
\hline \multirow{2}{*}{ Maternal education } & Unable to read and write & 20 & 12 & 1.86 & $0.35-9.82$ \\
\hline & Able to read and write & 102 & 110 & 1 & \\
\hline \multirow{2}{*}{ Monthly income less than 850} & Yes & 65 & 47 & 1.7 & $1.02-3.51^{*}$ \\
\hline & No & 57 & 75 & 1 & \\
\hline
\end{tabular}

*Significant associations.

\section{Discussion}

The aim of the present study was to identify factors associated with occurrence of diarrhoea, and pneumonia among children aged 7 to 24 months. To better see the role of exclusive breast feeding in the prevention of diarrheal, and pneumonia, cases and controls were compared.

The present study revealed that children with history of low birth weight, pre-lacteal feeding, and not exclusively breast fed were 12, 16, and 83 times more likely to be cases. Moreover, children born to mothers whose 
monthly income were less than 850 Birr were nearly two times more likely to be cases than those whose mothers' monthly income was higher than 850 Birr. However, in this study, educational status of mothers falls below the level of statistical significance.

Low birth weight one of the determinant factors for experiencing repeated attack of diarrhea, and pneumonia in the present study. This could be partly explained by the fact that low birth weight babies do have compromised immune system which makes them liable to different kinds of infections including pneumonia, and diarrhea.

In the present study, the proportion of pre-lacteal feeding among cases, and controls was $87 \%, 17 \%$, respectively, and hence those children who exposed for pre-lacteal feeding before six months of age were 16 times more likely to experience diarrhoea or pneumonia. This finding is in agreement with international recommendations [7] [8]. The use of prelacteal feeds is not recommended because it can make the infant ill which in turn interferes with breastfeeding [7].

Exclusive breast feeding is an internationally recommended strategy to prevent diarrhoea, and pneumonia in children. Unfortunately, however, in the present study, overall prevalence of exclusive breast feeding was only $52 \%$ (17\% among cases, and $87 \%$ among controls). Although, the overall prevalence of exclusive breast feeding seems to agree with international target, and Ethiopia is one of the countries which achieve this target [9], the prevalence of exclusive breast feeding among the cases was only $17 \%$. Hence, in the present, those children who had no chance to be beast fed were 83 times more likely to be cases than those who were exclusively breast fed.

According to International Vaccine Access Centre (2013) [9], Exclusive breast feeding is especially critical to reducing young children's mortality and morbidity from pneumonia, and diarrhea in countries with poor water, sanitation, and hygiene, exclusive breastfeeding.

According Lauer et al. (2006) [10] to the size of the gap between current practice (low level of exclusive feeding) and recommendations is striking when one considers breast-feeding involves no out-of-pocket costs and that implementing current international recommendations could potentially save 1.45 million children's lives each year.

This study could have been better if the design of the study were cohort study design instead of case control because case control study is inappropriate for common events or diseases such as pneumonia, and diarrheal diseases for odds ratio as measure of strength of association in common events could be very much inflated (up to $90 \%$ ) [11]. Therefore, an AOR of 83 (association between exclusive breast feeding and occurrence diarrhoea and pneumonia) could be equivalent to AOR of 8 , It still heralds a very strong association. It should also be remembered that case control study design has the following advantages over cohort study design: cheap, can be done in short period of time and no lose to follow up.

\section{Conclusion}

Eexclusive breastfeeding in the first six months of infant life is an important element in preventing to diarrheal and pneumonia in our setup. Hence, mothers should be encouraged to exclusively breast feed their infants in our, and similar set ups where access to potable water, and sanitation and hygiene is still a challenge.

\section{Acknowledgements}

We are very grateful to the Bahir Dar University for the approval of the ethical clearance and for their technical and financial support of this study. Then, we would like to thank all women who participated in this study for their commitment in responding to our interviews.

\section{Competing Interests}

The authors declare that they have no competing interests.

\section{Authors' Contributions}

RB wrote the proposal, participated in data collection, analyzed the data and drafted the paper. MG approved the proposal with revisions, participated in data analysis and revised subsequent drafts of the paper. All authors read and approved the final manuscript. 


\section{References}

[1] Horta, B.L. and Victora, C.G. (2013) A Systematic Review on the Benefits of Breastfeeding on Diarrhoea and Pneumonia Mortality. World Health Organization.

[2] UNICEF (2011) Lack of Optimal Breast Feeding in Developing Countries.

[3] Maheswari, E., Vishnu, B. and Mohamed, A. (2010) Knowledge, Attitude and Practice of Breast Feeding among Postnatal Mothers. Current Pediatric Research, 14, 119-124.

[4] César, U.A., Victora, C.G., Barros, F.C., Santos, I.S. and Flores, J.A. (1999) Impact of Breastfeeding on Admission for Pneumonia during Post Neonatal Period in Brazil. BMJ, 318, 1316-1320. http://dx.doi.org/10.1136/bmj.318.7194.1316

[5] (2011) Ethiopian Demographic and Health Survey. Central Statistics Authority, Amhara Region.

[6] Victor, C.G., Kirkwood, B.R., Ashworth, A., Black, R.E., Rogers, S., Sazawal, S., Campbell, H. and Gove, S. (2012) Potential Interventions for the Prevention of Childhood Pneumonia in Developing Countries: Improving Nutrition.

[7] (2004) Breast Feeding-Practices, Problems and Prospects.

[8] Ketsela, T. and Kebede, D. (1996) Pattern of Feeding Infants in Addis Ababa. Ethiopian Journal of Health Development, 10, 133-143.

[9] International Vaccine Access Center (IVAC) (2013) Pneumonia and Diarrhea Progress Report.

[10] Lauer, J.A., Betran, A.P., Barros, A.J.D. and de Onis, M. (2006) Deaths and Years of Life Lost Due to Suboptimal Breast-Feeding among Children in the Developing World: A Global Ecological Risk Assessment. Public Health Nutrition, 9, 673-685. http://dx.doi.org/10.1079/PHN2005891

[11] Osborn, J.W. (2006) Bringing Balance and Technical Accuracy to Reporting Odds Ratio and the Results of Logistic Regression Analysis. Practical Assessment Research and Evaluation, 11, 1-6. 